\title{
Printer control using Mobile
}

\author{
Narayan N.Dhage, Dr.S.D.Markande \\ Department of Electronics and Telecommunication NBN Sinhgad school of Engineering ,Pune Pune,India \\ Department of Electronics and Telecommunication NBN Sinhgad school of Engineering ,Pune Pune,India
}

\begin{abstract}
The common data printing procedure has to use notebook or desktop computer as a relay medium, first receiving the data from mobile and then sending it to printer using an appropriate printer. This is rather cumbersome. This paper proposes a design of a device which directly print the data stored inside the Mobile without the assistance of a notebook or desktop computer.
\end{abstract}

Index Terms: Notebook computer, Desktop computer, Printer

\section{INTRODUCTION}

An adaptor which mediates between a mobile device and a printer causes the printer to print by selecting either of the first operation mode in which data from the data supply device is received by wireless communication and before the completion of receiving the data, a print job is issued to the printer by wired communication, and the second operation mode in which data from the supply device is received by wireless communication and after the completion of receiving the data, a print job is issued to the printer by wired communication.

A printing system which prints by outputting, to a printer via an adaptor, data held by an data supply device, the adaptor, comprising a first interface configured to communicate with the printer by a first communication protocol, and a second interface configured to communicate with the data supply device by a second communication protocol different from the first communication protocol; and the printing system being configured to operate in a first operation mode in which image data from the data supply device is received by the second interface, and before completion of receiving the data by the second interface, a print job for printing data that is received before the completion of receiving the data by the second interface is issued from the adaptor to the printer via the first interface; and a second operation mode in which image data from the image supply device is received by the second interface, and after completion of receiving the image data by the second interface, a print job for printing image data whose reception has been completed by the second interface is issued from the adaptor to the printer via the first interface; the printing system comprising: a selection unit configured to select either of the first operation mode and the second operation mode in which the adaptor issues the print job; and a switching unit configured to switch between the first operation mode and the second operation mode in accordance with a selection result of the selection unit, wherein the selection unit selects either the first operation mode or the second operation mode based on at least one of a file name, an image format type, a transfer time and accessory information of the image data received from the image supply device.

\section{LITERATURE SURVEY}

The present day advancement in technology before us is a result of chain of pain staking research and improvisation process. It is reflected as "sophistication" everywhere - whether it is office automation, household appliances, multimedia and entertainment gadgets or medical equipment where "connectivity" and "touch of a button" ensures the compliance of desired process. This "sophistication" is because of the "convergence" of technologies [2].

Technology development should help people to do their work more easily and make them comfortable [1]. Printer control using Mobile device is one of the Useful technology that will help people in their day to day life. The conventional printer requires Mobile as input device, LAN connection and the printer as an output device. The idea of using Mobile phone to printing data using wireless technology. Mobile phone should contain Bluetooth through which user can able to print data [3].

\section{Problem Statement}

The proposed system aims at providing a means to directly send data stored in Cell phone to printing device for printing without using Computer. Sometimes it is not possible to have internet but nowadays everyone has mobile internet facility if we can directly give printing from mobile then it minimizes efforts. The reason is that one has to use a notebook or desktop computer as a relay medium, receiving the data from an mobile and then sending it to a printer using an appropriate printer driver. This is rather cumbersome. That is, it is impossible 
to directly print the data stored inside the mobile without the assistance of a notebook or desktop computer. The input to the system is data file to print and the output of the system is serial data in printing format.

\section{OBJECTIVE}

- Wirelessly print documents, bills and invoices (MS Office: Word, Excel, PowerPoint; PDF and text files) from device memory, SD card and even from Google Drive (Google Docs).

- Instantly print emails from Gmail, print photos and images (JPG, PNG, GIF), also print contacts, agenda, sms/mms, call log, web pages (HTML) and other digital content directly from your device to a printer right next to you or anywhere in the world. simply needs to be on the device to unlock Premium Features. Prior to buying the key we highly recommend printing the test page to ensure compatibility with your printer.

\section{SCOPE OF Proposed System}

- $\quad$ Proposed system is for printing directly from cell phone .

- The Laptop/Computer users wishing to have their document to be print would have to connect to a local printer, has to install print devices drivers which can be reduce by using proposed device.

- This approach can be used for time saving and it is simply practical. It is easier to print data from mobile device like Mobile Phones, Laptops, PCs etc. which can send data via Bluetooth.

\section{A. Wireless connectivity between Mobile and Printer-}

\section{METHODOLOGY}

Figure 1 shows the block diagram of complete architecture of BT Adapter .The BT module which may be $2.4 \mathrm{GHz}$ wireless module which acts as a BT Receiver. The BT module receives the signal from mobile and gives it to the controller. The memory unit of controller saves the data temporarily and gives it to the extraction unit .The extraction unit retrieve information and the compiling unit convert it into printing format . Through USB cables it is given to the printer for printing.

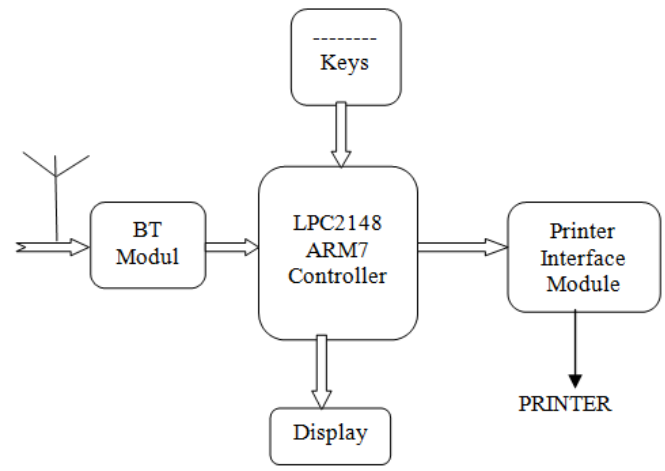

Figure 1. System block diagram

\section{B. The Reason for using ARM7(LPC2148)}

The ARM processor is currently used in industrial control, consumer electronics projects and network system and has a market share for 32-bit processors for RISC of over $75 \%$.

The LPC2148 processor chip produced by PHILIPS has a speed of $72 \mathrm{MHz}$ and some appropriate features of being used in BT to USB converter, which is with two USB 2.0 interfaces and BT can be interfaced.

\section{DETAILED DESCRIPTION}

The system structure is shown in FIG. 1. The disclosed BT/USB printing converter can receive data transmitted from a Bluetooth electronic product. The data can be directly sent to a printing device for printing Without needing any computer device. In the following, We explain the main components and relevant devices . (1) Bluetooth transmission module It is used to receive image data transmitted from Bluetooth electronic product such as mobile phones and PDA's.

(2) Microprocessor. It is connected to the Bluetooth transmission module and contains an extraction unit, a memory unit and a compiling unit. The memory unit temporarily stores the image data for subsequent uses. The extraction unit extracts the image information to be printed from the image data transmitted by the Bluetooth electronic product. The image information to be printed can be in the OPP formats. The compiling unit converts the image information extracted from the image data into printing data according to the picture bridge protocol. The picture bridge protocol is a printing protocol development in the industry in recent years. It enables the data transmissions among different types of electronic products and printing devices. 
(3) USB transmission module. It is connected to the microprocessor and in electrical communications With the USB of the printing device. It can transmit the printing data provided by the microprocessor to the printing device for printing.

(4) Protection unit. It is electrically coupled to the microprocessor and the USB transmission module. It is used to prevent the USB transmission module from transmitting too much electric power to the microprocessor to cause damages. A BT/USB printing conversion method, After each printing element in the printing device is confirmed to return its initial position, the data transmission mode of the printing device is initialized to get ready for receiving external information. The Bluetooth transmission module continues to detect the data transmission status of the electronic product. Once the electronic product starts to transmit data, the system receives data transmitted from the electronic product by using the Bluetooth transmission function and temporarily stores the data in the memory unit. Afterwards, the system detects the format of the data . If the data reception is incomplete or the format is incorrect, the system returns to receive another set of data or request new transmissions. If the format of the data is correct, the extraction unit extracts the information to be printed from the data the system converts the image information to be printed into printing data according to the picture bridge protocol .The picture bridge protocol is a printing protocol development in the industry in recent years. It enables the data transmissions among different types of electronic products and printing devices. The system printing task starts after the system transmits the received printing data to the printing device. Finally, the system checks Whether another set of image data are received .If so, the system goes back otherwise, the Whole procedure ends. The disclosed BT/USB image-printing converter and the associated method can solve the problems encountered in the prior art, Where one has to rely on the assistance of a computer device and proper installation of a printer driver in order to transmit image data from Bluetooth electronics to a printer. Using this method, the data can be directly transmitted from a Bluetooth electronic product, such as the mobile phone, digital camera, and PDA, to a printing device for printing Without the need of a computer.

\section{CONCLUSION}

This study proposed the design of a low cost Bluetooth to USB converter, which is capable of receiving data and controlling Printer device via the Bluetooth port of Mobile

\section{REFERENCES}

[1] Stefan Nowak, Falk-Moritz Schaefer "Towards a Convergent Digital Home Network Infrastructure" IEEE Transactions on Consumer Electronics, pp. 1695-1703, Nov.2011.

[2] Olawuyi J.O. Mgbole Friday, "Technological Convergence" Science Journal of Physics, pp.7237-7242, 2012.

[3] Amarnath M, "Home appliance control using mobile cloud technology" Proceedings of International Conference on Modeling optimisation and computing, pp. 3587-3595, 2012. 